\title{
Cruce de centenarios: la utopía anarquista de Pierre Quiroule
}

\author{
Celia de Aldama OrdóÑEZ \\ Departamento de Filología Española IV. Literatura Hispanoamericana \\ Universidad Complutense de Madrid \\ c.aldama@ucm.es
}

Recibido: $14 / 10 / 2014$

Modificado: 22/05/2015

Aceptado: 27/05/2015

\section{Resumen $^{1}$}

Este ensayo explora la encrucijada entre dos modelos urbanos; por un lado, el modelo utópico detallado en la obra literaria de Pierre Quiroule La ciudad anarquista americana, y, por otro, la ciudad de Buenos Aires, que celebra en 1910 el Centenario de su Independencia. La ficción de Quiroule, que propone la fundación de su urbe ideal sobre las ruinas de la monarquía de El Dorado, es una de las voces que, desde las filas libertarias, se levantan contra el paradigma ideológico defendido por la oligarquía criolla en la primera década del siglo XX.

Palabras clave: Pierre Quiroule, ciudad, utopía, anarquismo, Centenario argentino.

Title: Intersection of Centenaries: the Anarchistic Utopia of Pierre Quiroule

\section{Abstract}

This essay explores the crossroads between two models of city. First, the Utopian model detailed by Pierre Quiroule in his work La ciudad anarquista americana, and secondly the city of Buenos Aires, which celebrates the Centenary of its Independence in 1910. The fiction of Quiroule, which proposes the foundation of its ideal city over the ruins of the monarchy of El Dorado, represents one of the voices of Argentinian anarchism that rise up against the nationalist discourse supported by the Creole oligarchy in the first decade of the $20^{\text {th }}$ century.

Keywords: Pierre Quiroule, city, Utopia, anarchism, Argentine Centenary.

\section{Índice}

1. Introducción

2. La Ciudad Ideal

3. Derivas textuales: de la utopía literaria a la comuna libertaria

4. A modo de conclusión

${ }^{1}$ Este artículo se inscribe en el marco de la investigación doctoral llevada a cabo con una beca FPU concedida por el Ministerio de Educación, Cultura y Deporte. 
iNo; no queremos más de vuestro estupendo progreso, hombres de genio! iNi de vuestras colosales ciudades, ilustres patriotas! iEstas grandes ciudades, cuyo esplendor amasado con sangre de proletarios, esconden tantas lacras asquerosas! iQueremos luz, queremos aire, queremos sol... y en vuestras ciudades y en vuestra organización social solo hay asfixia y tinieblas!

(Pierre Quiroule)

\section{Introducción}

La primera evidencia que se desprende de la obra de Pierre Quiroule ${ }^{2}$, La ciudad anarquista americana, es su estructura dialéctica al ser su andamiaje literario resultado de una colisión, de un encuentro problemático entre dos modelos de ciudad. La utopía engendrada en el texto quirouliano releva las fracturas de la época histórica en que radica $y$, desde los espacios ficcionales que en ella se despliegan, se problematizan los contornos, impuestos por la élite criolla, para la ciudadanía argentina. Con mirada retrospectiva, este análisis propone, a partir de dicha obra, publicada en el año 1914, deshilvanar el curso histórico en que se gesta $y$, desde el presente utópico de su escritura, retrotraerse hasta el mes de mayo de 1910 en que la nación celebra el Centenario de su Independencia.

El texto de Quiroule ${ }^{3}$, cuya longevidad de cien años se ha querido conmemorar con este ensayo, ha de leerse, por tanto, en relación al convulsionado cuadro sociopolítico que lo contiene y explica; la utopía urbana que la obra imagina no puede entenderse sino como reacción, ataque y negación del modelo de "ciudad moderna" que, desde los sectores oligárquicos ${ }^{4}$, se proyecta como arquetipo para la joven nación americana.

2 Entre los datos biográficos del autor, resultan de particular interés para este estudio su condición de inmigrante y su incorporación a los grupos anarcocomunistas porteños, corriente ideológica que prevalece en los círculos anarquistas argentinos a finales de siglo (Gómez Tovar et al. 1991: 46). Pierre Quiroule (18671938) nace en Lyon y llega en los primeros años de su infancia a Buenos Aires, donde trabaja como tipógrafo de la Biblioteca Nacional y como colaborador de La Protesta, periódico anarquista que publica la obra que aquí se estudia.

3 Félix Weinberg (1976: 105) señala que la obra forma parte de "un tríptico de utopías en las que Quiroule vislumbra una nueva sociedad liberada de todo género de opresión". Además de La ciudad anarquista americana, el autor publica Sobre la ruta de la anarquía (1912) y En la soñada tierra del ideal (1924).

${ }^{4} \mathrm{Si}$ bien no es posible hablar de consenso entre las elites acerca del modelo de ciudad moderna, la mayoría de las propuestas que se formulan desde los sectores hegemónicos coindicen en privilegiar la componente industrial de la capital bonaerense y advierten de una necesaria nacionalización de la masa heterogénea que la habita. 


\section{La Ciudad Ideal}

La Ciudad de los Hijos del Sol reemplaza, en la ficción literaria de Quiroule, a la monarquía de El Dorado y su cartografía imaginaria se concibe como el resultado efectivo de la revolución anarquista; la "emancipación del proletariado" y la "desaparición de la dominación burguesa" (Quiroule 1991: 17) constituyen los baluartes del nuevo orden social, al que el lector accede a través de la forma urbana, pues ésta constituye su "transparencia" (Ansolabehere 2001: 271) ${ }^{5}$. La planta citadina, detallada por el autor en un mapa que adjunta al relato (figura 1), constituye la prueba material de la armonía que reina en la comuna anarquista, cuyos habitantes, ajenos a las perversiones del mundo capitalista, conviven en el ambiente de confraternidad y "ayuda mutua" teorizadas por el anarco-comunista ruso Kropotkin. La ciudad de los Hijos del Sol alberga a un máximo de doce mil habitantes y su ubicación se localiza en un "claro y alegre paisaje" (Quiroule 1991: 17); filas de viñedos y arroyos de agua cristalina la engarzan en un terreno fértil, quedando el tópico clásico del locus amoenus conciliado con el idílico contexto ciudadano imaginado por Quiroule ${ }^{6}$.

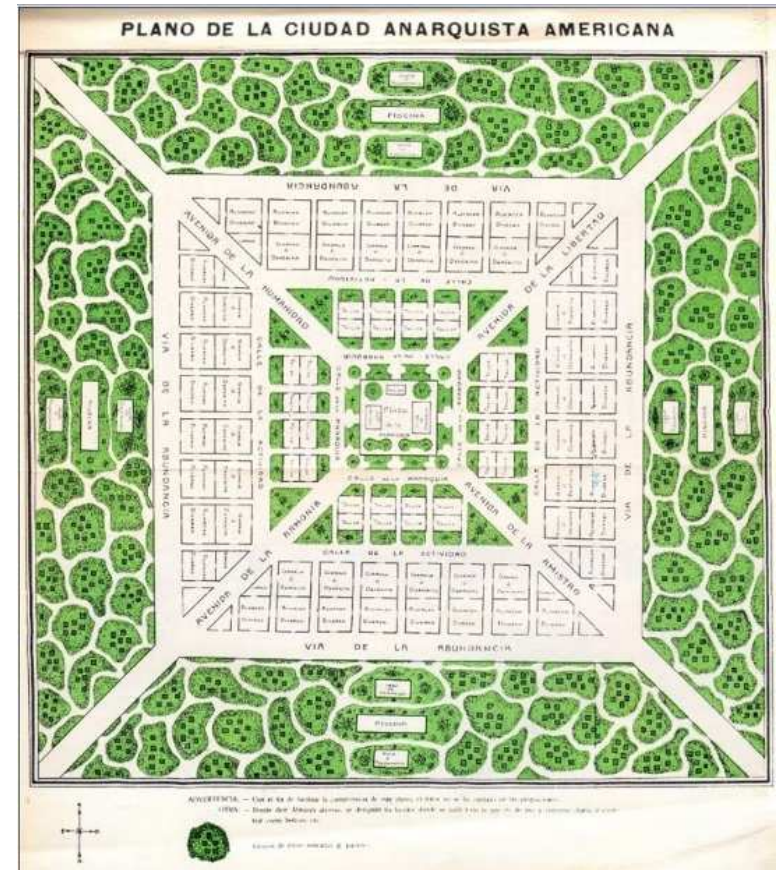

Figura 1. Plano de Las Delicias.

${ }^{5}$ Pablo Ansolabehere (2011: 271) intuye la existencia de una relación directa entre forma urbana y estructura social, concluyendo que la "hipótesis de La ciudad anarquista americana es que a cada orden social le corresponde un tipo determinado de ciudad".

${ }^{6}$ La armoniosa integración en la red citadina de zonas verdes y áreas de trabajo queda reflejada en el susodicho mapa que, entendido como pieza funcional de la narración, reincide en la disposición de las estructuras urbanas, las jerarquías que las ordenan y la ideología que subyace a su distribución en el conjunto topográfico. 
La plaza, inspirada quizás en las ágoras de la Grecia Antigua, ocupa el enclave central de la ciudad, y en ella confluyen las cuatro avenidas principales: Libertad, Amistad, Armonía y Humanidad. En su espacio abierto, ocupado por el Teatro, el Hall de Gimnasia y la Sala del Consejo, todos ellos concebidos como lugares de sociabilidad y no de gobierno, se reúnen los Hijos del Sol para resolver las cuestiones que atañen a la vida de la comunidad. Los talleres, almacenes y depósitos se distribuyen en hileras cuadrangulares, formando una retícula urbana de estructura en damero. Las áreas residenciales, conformadas por pequeños grupos de casas rodeadas de jardines, se sitúan en la periferia, cerca de las piscinas y los centros educativos, y lejanas del bullicio del centro. De tal panorámica se eleva la silueta de la ciudad que Quiroule propone como alegoría y encarnación del ideal anarquista; es la ciudad futura destinada a reemplazar a la gran metrópolis contemporánea.

La ciudad de los hijos del Sol se construye a lo largo del relato utópico no como elemento insular, sino como término de oposición frente a la ciudad de Las Delicias, que representa, en el contexto de la obra, su exemplum ex contrario. Ambas ciudades reproducen modelos excluyentes y su incompatibilidad resulta intrínseca a cada uno de los elementos que animan su disposición urbana y espiritual. Si el trazo de la primera corresponde al patrón de una pequeña comunidad autogestionada y administrada por un sistema colectivista, el de la segunda impone el modelo de ciudad-fábrica, sumida en un vórtice de industrialización y atestada de personas, residuos y epidemias ${ }^{7}$. Las Delicias constituye el hábitat natural del parásito burgués, donde confluye "todo lo que puede dañar y perjudicar al hombre: suciedad, enfermedad, corrupción, delincuencia, opresión y esclavitud" (Quiroule 1991: 146).

En la ficción de Quiroule, la ola revolucionaria arrasa la capital burguesa y la victoria sobre ésta coincide con la celebración del décimo aniversario de la monarquía del Dorado $^{8}$ : los libertarios raptan al rey y a sus ministros y, en procesión apocalíptica, marchan sobre las ruinas de la ciudad. El triunfo del sistema anarquista sobre la metrópolis moderna radica no sólo en la destrucción de su adversaria, sino en el ejemplar funcionamiento de la Ciudad de los

\footnotetext{
7 Mientras los referentes del modelo utópico son rastreables en las propuestas socialistas de Owen, San Simon y Fourier, además de en las fuentes clásicas de Tomás Moro y Domenico Campanella, los de la segunda reproducen el escenario distópico de la ciudad capitalista, en auge en Occidente tras el desarrollo industrial. ${ }^{8}$ Como se verá en el siguiente epígrafe, la elección de la fecha no es casual sino que sirve a Quiroule para entrelazar su relato con los episodios nacionales recientes $y$, en concreto, con los festejos conmemorativos de 1910, celebrados bajo el estado de sitio por el riesgo de ofensivas anarquistas.
} 
Hijos del Sol, cuyo impecable engranaje parece garantizar su exitosa extrapolación al mundo no ficcional.

\section{Derivas textuales: de la utopía literaria a la comuna libertaria}

En este segundo momento de la reflexión, la propuesta textual de Quiroule se manejará como pantalla a través de la que reconfigurar las tensiones que saturan el conjunto nacional argentino entre 1900 y 1910. A partir del sistema binario que el autor deriva de la oposición entre "ciudad revolucionaria" y "ciudad burguesa", se intentará visibilizar la línea de fuga que lo vincula directamente con la ciudad de Buenos Aires $y$, en consecuencia, con el que José Luis Romero define como el Espíritu del Centenario ${ }^{9}$.

La crítica ha apuntado las coincidencias que esclarecen tal referencialidad ${ }^{10}$, demostrando que Las Delicias no es más que un trasunto literario de la capital bonaerense. El propósito de este análisis, en cambio, no es el de reconstruir el referente porteño a través de las analogías diseminadas por Quiroule, sino estudiar las oposiciones que el autor establece entre éste y la Ciudad de Los Hijos del Sol. Al cotejar ambas ciudades, la ideal y la real, se demostrará que la primera incorpora, en negativo, la cartografía de la segunda y que tal operación conceptual es implementada como herramienta de praxis política, es decir como vía de problematización y de intervención en el presente de la vida nacional.

Dada la meticulosidad y extensión de la propuesta de Quiroule, se referirán tres aspectos definitorios de la ciudad ácrata, que evocan, a su vez, tres núcleos conflictivos con respecto a su contraria. Si los proyectos de la elite criolla apuntan a convertir Buenos Aires en una ciudad oligárquica, nacionalista e industrializada, Quiroule defiende un modelo anarquista e internacionalista que

9 En su obra El desarrollo de las ideas de la Argentina del siglo XX, José Luis Romero (1983) identifica el "Espíritu del Centenario" con una serie de iniciativas reformistas que, en la primera década del siglo XX, son debatidas por una nueva generación de políticos e intelectuales. Entre los tópicos que conforman la red discursiva de dicho periodo, en el que se hace un balance de la vida nacional, destacan la inmigración, la salud pública o la seguridad nacional.

10 Me interesa particularmente el análisis de Pablo Ansolabehere en el que se apuntan algunos localismos que denuncian la argentinización de la ciudad anarquista americana. Entre los elementos que señala para demostrar la correspondencia entre Las Delicias y Buenos Aires, resultan evocadoras las alusiones a la pampa, al parcelamiento de la tierra, al gaucho y a los festejos patrióticos. Quiroule, señala el crítico, es testigo de los cambios modernizantes que afectan a la ciudad porteña desde su capitalización y que hacen de la gran aldea una metrópolis incontrolable, una "ciudad efímera" (Ansolabehere 2011: 281). Asimismo, el estudio de la toponimia realizado por Félix Weinberg concluye que Quiroule "sitúa la región de El Dorado en el ámbito bonaerense y su adyacencia, el litoral argentino" (Weinberg 1976: 70). 
privilegie el trabajo agrícola y artesanal. A continuación, se observará en detalle cómo estas oposiciones cristalizan los antagonismos entre ambas ciudades, que al fin y al cabo traducen, en terminología de Althusser, distintos aparatos ideológicos ${ }^{11}$.

El antagonismo más evidente entre la Ciudad de los Hijos del Sol y Buenos Aires estriba en las formas políticas que las gobiernan. En la urbe anarquista fantaseada por Quiroule, los lazos de solidaridad que median entre sus habitantes cohesionan el tejido urbano garantizando la paz social: los Hijos del Sol no delinquen ni contravienen el orden citadino ${ }^{12}$. Tal espectáculo de bonhomía acentúa las convulsiones radicadas en el escenario porteño que, a comienzos de la centuria, vive en un permanente estado de sitio; huelgas, protestas armadas y atentados anarquistas evidencian las grietas que socavan el pacto social de la Argentina liberal ${ }^{13}$. Frente a la civilizada ciudad de la revolución, Buenos Aires se proyecta, más allá del contexto ficcional creado por Quiroule, como receptáculo de barbarie; la oligarquía criolla, parapetada en el poder gracias a continuados fraudes electorales, cierra cada vez más sus filas y no hesita en promulgar decretos legislativos ${ }^{14}$ que expulsen de las fronteras de la patria a los agentes patógenos ${ }^{15}$ que la amenazan. La maqueta de Quiroule problematiza, desde la amenidad del relato

11 Los aparatos ideológicos del Estado, señala Althusser, están formados por instituciones distintas como las religiosas, familiares, escolares, políticas o culturales y éstas, añade, "pueden no solo ser objeto sino también lugar de la lucha de clases" (Althusser 1988: 28).

12 "El cambio de forma social había operado una transformación radical en la mentalidad general. La abolición de la propiedad privada y la supresión del oro como valor representativo de la producción, había asestado un golpe mortal a la delincuencia" (Quiroule 1991: 45).

13 McGann (1960: 381) destaca el clima de incrementada violencia que se vive en Argentina entre 1902 y 1910 , años en que el pueblo argentino conoció cinco veces el estado de sitio. Entre los altercados más relevantes, han de destacarse la primera huelga general (1902), la protesta armada del Partido Radical (1905), el asesinato del coronel Falcón (1909) y la bomba en el Teatro Colón (1910).

${ }^{14}$ La Ley de Residencia, propuesta por el político y escritor argentino Miguel Cané y sancionada por el gobierno de la Nación en 1902, habilitaba en su Artículo II a expulsar a "todo extranjero cuya conducta pueda comprometer la seguridad nacional" (Cané 1899: 3). La iniciativa, que ha de entenderse como parte del programa de robustecimiento del aparato legislativo, se justificaba como medida de protección del cuerpo nacional y es síntoma del acorazamiento de la oligarquía criolla ante la pérdida del control de la República Argentina.

${ }^{15}$ Esta idea de la nación como cuerpo vivo, cuyos obstáculos han de ser erradicados como enfermedades o gérmenes patológicos, es señalada por Óscar Terán (1987: 85) como indicio de la asimilación de la filosofía positivista, que "alcanzó en Argentina una penetración imposible de subestimar, ofreciéndose como organizador fundamental de la problemática político-social de la élite entre el 90 y el Centenario". 
utópico, los resortes de un aparato estatal defectuoso, cuyas formas conservadoras de gobierno quedan deslegitimadas ante un modelo anarquista garante de la concordia urbana.

Por otra parte, la escritura de Quiroule emerge como refutación a una discursividad estigmatizadora que, puesta en circulación por los representantes de la élite político-intelectual y fundamentada en los postulados científicos de Cesare Lombroso ${ }^{16}$, establecía redes de inmanencia entre anarquismo y criminalidad. Citaré, a modo de ejemplo, las declaraciones de dos hombres de Estado; las siguientes palabras de Estanislao Zeballos, "hay en el mundo un grupo de hombres que hace gala de no tener Dios, ni Patria, ni Ley. Su principio y su fin está en el ejercicio de la violencia y del crimen" (Botana y Gallo 1997: 523), corroboran dicha equivalencia, rastreable también en los discursos de Lucas de Ayarragaray, para el que el anarquismo constituye "un delito contrario a la civilización argentina" (Botana y Gallo 1997: 528) ${ }^{17}$. Del conglomerado discursivo que nutre tal proceso de criminalización, Ansolabehere destaca, además del texto de Lombroso, varios casos argentinos ${ }^{18}$, demostrando que la correlación entre lo anarquista y lo terrorista, en la Argentina de entresiglos, es rastreable en expresiones culturales de toda índole, desde el sainete criollo hasta el ensayo de interpretación nacional ${ }^{19}$.

Como último apunte para demostrar cómo desde el campo de poder se instituyó un proceso de estigmatización biológica del anarquista, citaré las Notas de filosofía penal del ministro argentino Cornelio Moyano Gacitúa que, con terminología naturalista, describe

16 Las obras criminológicas de Lombroso gozan de notable difusión en Argentina que, desde la década del ochenta, se enfrenta a la irrupción, cada vez más impetuosa, de la ideología libertaria. En su tratado Gli anarchici, se documenta la estrecha vinculación entre anarquismo y delincuencia, y se proponen una serie de medidas profilácticas, cuales el exilio y la deportación, para combatir a los secuaces del ideario ácrata (Lombroso 1895).

17 Frente a las explicaciones tradicionales que atribuían el arraigo del anarquismo en Argentina al alto porcentaje de inmigrantes españoles e italianos, Ricardo Falcón (1986-1987) añade tres causas alternativas: el régimen político, que marginaba del poder a amplios sectores de la población, la cuestión social, relacionada con las reivindicaciones laborales y, por último, la cuestión nacionalista, a la que se aludirá en el siguiente punto de este estudio.

18 Ansolabehere (2011: 545) estudia el "fenómeno de ósmosis discursiva que se verifica entre la literatura científica (en especial la criminológica) y la ficción" y se concentra en el análisis de la figura del anarquista delincuente en el Libro extraño de Sicardi y el proyecto de ley de Miguel Cané.

19 Por el contrario, en la utopía quirouliana, el personaje de Súper representa al hombre de ciencia que, poniéndose al servicio de la revolución, sugiere ese desplazamiento del estereotipo del anarquista como delincuente al modelo del anarquista como héroe constructivista que emplea sus conocimientos científicos para pensar otro mundo posible. 
el ideario ácrata como "agente mórbido" y "virus maléfico"20. La monstruosidad del anarquista radica en su naturaleza híbrida, que resulta incatalogable para la ciencia médica:

No son locos y obran como locos, son todo y nada, mezcla informe de delirio y de razón, de fatalismo y voluntad, escapan a la clasificación científica: son monstruos como diría Tarde. Son monstruos sociales, pero su procedencia más próxima parece estar en esas nebulosidades filosóficas que tampoco son sombra ni luz. (Moyano Gacitúa 1894: 19)

El segundo aspecto de la realidad argentina que Quiroule cuestiona es el relativo al inmigrante europeo que, atraído por las campañas de repoblación nacional ${ }^{21}$, llegó a superar al nativo durante varias décadas ${ }^{22}$. Los debates que, alrededor de 1910 y en un contexto de incipiente nacionalismo ${ }^{23}$, desata la figura del extranjero pueden leerse en el texto de Quiroule, entendido una vez más como término de oposición. La Ciudad de los Hijos del Sol es fiel a los principios internacionalistas del ideario ácrata; el territorio argentino es concebido, por tanto, como parte de una "América colosal y magnífica", comprometida con la redención de las "razas esclavizadas de Europa", y liberadora de las "últimas cadenas del género humano" (Quiroule 1991: 105). La noción de lo social, en cuanto factor extensible y comprehensivo, contradice los presupuestos biologicistas que, en la Argentina real, justificaron la persecución de indios y de

20 "Nuestra civilización está combatida por agentes mórbidos que lleva en sus entrañas, y que como tales agentes mórbidos, difieren de su falta de desarrollo evolutivo. Los defectos de nuestro régimen son notorios aunque hijos, más que todo, de nuestro estadio evolutivo" (Moyano Gacitúa 1894: 13).

${ }^{21}$ Sarmiento y Alberdi promovieron la activación de medidas migratorias con el fin de aumentar los índices demográficos del país y poblar los espacios vacíos del desierto pampeano con mano de obra europea. La Constitución de 1853 constituye un nítido reflejo del pensamiento de esa generación y establece que el Gobierno federal "no podrá restringir, limitar ni gravar con impuesto alguno la entrada en el territorio argentino de los extranjeros que traigan por objeto labrar la tierra, mejorar las industrias, e introducir y enseñar las ciencias y las artes" (Constitución de la Nación Argentina 2008: Art. 25).

22 "El máximo anual (de inmigrantes) fue alcanzado en la primera década del siglo (112.000 de promedio) y en particular en los años inmediatamente anteriores a la primera guerra mundial, que registró el año máximo con un saldo en la inmigración de ultramar de más de 200.000 personas" (Germani 1979: 244).

23 "En la forma de la inmigración masiva, desbordante hacia fin de siglo, la otredad despierta el mecanismo complementario de la autoafirmación, lo que se traduce en términos de identidad que, en el caso y el momento, se expresa mediante apelaciones esencialistas, muchas veces recortadas sobre gestos apenas disimulados de miedo al extranjero y con una exageración expresiva que prepara la llegada de las retóricas del nacionalismo" (Alfieri 2006: 515). 
gauchos, primero, y la criminalización y patologización del inmigrante ${ }^{24}$, después.

Quiroule muestra su aversión a toda forma de patriotismo al describir a los protagonistas de los festejos del aniversario de Las Delicias como "masas compactas y turbulentas, borrachas de patriótico entusiasmo y de aguardiente barato" (Quiroule 1991: 124). En estas líneas, es explícita la alusión a la Argentina del Centenario, cuyos contornos coindicen con un espacio infranqueable, cercado por los símbolos de una nación "inventada"25. Frente al espíritu de confraternidad que reina en la Ciudad de los Hijos del Sol, en el escenario porteño proliferan las deportaciones y los discursos xenófobos, que responsabilizan al extranjero de los males que afligen al cuerpo social. Dicho estigma encuentra sus antecedentes en la narrativa naturalista finisecular ${ }^{26}$ que, en vista del fracaso de los proyectos de europeización sarmentinos, resignifican los contenidos del "ideologema inmigrante": el extranjero pasa de ser agente de progreso y civilización a convertirse en el invasor bárbaro, un ser patógeno importador de teorías subversivas.

Será en la primera década del siglo cuando se producirá la codificación programática de los principios que inspiran el primer nacionalismo argentino, representado por una nueva generación de intelectuales, entre los que destacan las figuras tutelares de Ricardo Rojas, Manuel Gálvez y Leopoldo Lugones. Aunque sus propuestas revisionistas no suponen una homogeneidad ideológica, todas coinciden en su defensa del espíritu nacional frente al cosmopolitismo reinante, la restauración del "orden" frente a la "ciudad fenicia". Para ilustrar tal actitud, me referiré someramente a dos obras que, según Payá, representan la "expresión más sólida y acabada de los balbuceos nacionalistas de principios de siglo" (Payá y Cárdenas 1978): La Restauración nacionalista (1909) de Rojas y el Diario de Gabriel Quiroga (1910) de Gálvez. Si en la primera se pretende que el hijo del inmigrante sea "profundamente argentino", en la segunda se advierte de los peligros que la inmigración acarrea para la identidad nacional y se actualiza el lema alberdiano "gobernar es poblar" con la nueva fórmula "gobernar es argentinizar", de modo que la propuesta apátrida de Quiroule choca frontalmente con la

\footnotetext{
${ }^{24}$ Recuérdense, entre las acciones tomadas por la élite política para garantizar la "protección" y el "progreso" del Estado nacional, la Ley de Vagos, la Conquista del Desierto y, ya en el siglo XX, las Leyes de Residencia y de Defensa Social.

${ }^{25}$ Véase la reflexión de Shumway, incluida en su estudio La invención de Argentina, acerca de las tentativas de definición nacional para un Estado carente de una contundente demarcación simbólica. Asimismo, Altamirano y Sarlo (1997: 186) estudian los mitos de identificación colectiva que "habrán de dotar a los argentinos de una sólida tradición nacional frente a la amenaza de la invasión disolvente".

${ }^{26}$ Cambaceres, Argerich y Sicardi conforman los casos más representativos de este proceso de estigmatización.
} 
concepción esencialista de nación que, sustentada por políticas defensivas y excluyentes, se propone extirpar del suelo argentino todo indicio de heterogeneidad.

La tercera cuestión que enfrenta ambos modelos de ciudad es la relativa a las formas de productividad que rigen su economía. Si en las comunas de los Hijos del Sol se implanta un modelo alternativo de producción, basado en la autarquía, el progreso controlado y la tradición de las artes y los oficios, Buenos Aires, a partir de la fecha de su Centenario, exhibe una modernidad problemática, incompleta e ilusoria ${ }^{27}$. El habitante de la ciudad libertaria, nos dice Quiroule, sabe "indistintamente manejar un telar, imprimir un libro, hacer una instalación eléctrica, fabricar herramientas" (Quiroule 1991: 66). En la vida del porteño, en cambio, las rutinas mecánicas del oficio único sustituyen al trabajo "libre y variado" del sistema ácrata y, ante los episodios de accidentes y epidemias tan comunes en la capital bonaerense, los comunistas, asegura Quiroule (1991: 46), "viven en una ciudad limpia, alegre y sana, donde el aire es oxígeno puro, no un compuesto horrible de miasmas y podredumbres".

$\mathrm{Si}$ bien es cierto que Buenos Aires, en el centenario de la Revolución de Mayo, celebra entre sus triunfos el crecimiento económico, el desarrollo de sus vías ferroviarias y la importación de un completo sistema de comunicaciones ${ }^{28}$, la utopía quirouliana, con su ya conocida estrategia de contraste, discute el discurso oficial al evocar aquello que en este se oblitera. La insalubridad y la injusticia que caracterizan a los conventillos ${ }^{29}$, en la que Gino Germani

27 Entre los críticos y escritores que, desde encuadres teóricos divergentes, han cuestionado el proceso de modernización argentina, despunta el estudio de Beatriz Sarlo que definirá la cultura argentina de principios de siglo como "cultura de mezcla, donde coexisten elementos defensivos y residuales junto a programas renovadores" (Sarlo 1988: 18).

${ }^{28}$ Este clima de euforia es descrito por Osvaldo Pellettieri en la introducción de su labor de recopilación Testimonios culturales argentinos: la década del 10. "En pleno festejo del Centenario de la Revolución de Mayo, el país se hallaba en el pináculo de su poderío económico. Luego de largos años de penurias, y merced al famoso 'proyecto del ochenta', la Nación había logrado una casi increíble prosperidad de la cual se jactaban los gobernantes. Si bien resultaba evidente que la riqueza no estaba distribuida equitativamente, un optimismo casi total poseía, sobre todo, a los habitantes del litoral del Río de la Plata. Salvo una notoria minoría, compuesta por anarquistas y maximalistas, todo el mundo creía que la Argentina era un país dotado de las mayores gracias que Dios podía dispensar a los mortales. El progreso no iba a ser detenido por nadie" (Pellettieri 1980: 5).

29 Guillermo Rawson, primer catedrático de Higiene Pública, observa en sus Estudios sobre las casas de inquilinato en Buenos Aires "la degradación física y moral a que están sometidos estos habitantes en las condiciones de su albergue". Describe los conventillos como "fétidas pocilgas cuyo aire jamás se renueva y en cuyo ambiente se renuevan los gérmenes de las más terribles enfermedades" (Rawson s.f.: 3). 
denomina "época de transición"30, habrán de desaparecer, pues la ciudad ideal solo será viable en caso de fundarse ex nihilo, sobre un espacio y un tiempo primigenios.

\section{A modo de conclusión}

La narrativa de Pierre Quiroule no constituye un caso aislado en la Argentina de entresiglos, sino que se inserta en la red discursiva de adscripción anarquista ${ }^{31}$ que atraviesa su campo intelectual. Entre los colectivos ácratas, el texto literario deviene conducto de ideologías contra-hegemónicas y el ejercicio de la letra es entendido como continuum de la praxis política. En este contexto, la utopía de Quiroule, la invención de una arquitectura ideal, a la par que evidencia las exclusiones inherentes a la Argentina del Centenario, canaliza la expresión de su frustrada subjetividad política ${ }^{32}$; y es que el perfil del autor, debido a su problemática condición de anarquista e inmigrante, se enfrenta a un doble estigma que ubica su figura en el paradigma de lo invisible, lo excluido, lo marginado. Desde su posición periférica, desde el "afuera" al que lo relega la nueva noción de patria, Quiroule construye una ficción fundacional donde los sujetos non gratos del espectro nacional encuentran su representación. De la fricción que se genera, entonces, entre ambos modelos, la silueta porteña contrapuesta al falansterio quirouliano, se levanta una ciudad imaginada que pretende proyectar su Ideal más allá de la metáfora literaria que la contiene.

30 Gino Germani recompone el cuadro de transformaciones sociopolíticas que se producen en Argentina entre 1870 y 1910 y apunta que, en este periodo, "se cumple gran parte de la transición de la estructura tradicional hacia formas más avanzadas y más próximas a las sociedades industriales, por lo menos en lo que respecta a la zona metropolitana de Buenos Aires y a la región litoral" (1979: 263).

31 Entre los escritores anarquistas, Cappelletti (1990) destaca a Alberto Ghiraldo, Evaristo Carriego, José de Maturana y Rodolfo González Pacheco, entre otros. Junto a ellos, subraya la actividad de dos italianos: Errico Malatesta, fundador del Círculo de Estudios Sociales e importador de las primerias doctrinas anarquistas, y Pietro Gori, cuyo perfil intelectual atraerá a escritores y científicos argentinos. Para Ansolabehere (2011: 18), "lo que va a caracterizar al anarquismo argentino será el espacio privilegiado que va a reservarle a la actividad cultural. El sorprendente dinamismo editorial permite contar con títulos como El Perseguido o La Protesta, sin duda la publicación más importante de la historia del anarquismo latinoamericano". 32 La Ley de Defensa Social, aprobada el 27/06/1910, prohíbe "la asociación o reunión de personas que tenga por objeto la propagación de doctrinas anarquistas o la preparación o instigación a cometer hechos reprimidos por las Leyes de la Nación, y la autoridad local procederá a la disolución de las que se hubiesen formado e impedirá sus reuniones" (Ley de Defensa Social 1910: Art. 7, Cap. II). 


\section{Bibliografía}

ALFIERI, Teresa (2006): "La identidad nacional en el banquillo", en Noé Jitrik (dir.), Historia crítica de la literatura argentina, vol. V. Buenos Aires: Emecé.

ALTAMIRANO, Carlos; y SARLO, Beatriz (1997): Ensayos argentinos. De Sarmiento a la vanguardia. Buenos Aires: Ariel.

ALTHUSSER, Louis (1988): Ideología y aparatos ideológicos del Estado. Freud y Lacan. Buenos Aires: Ediciones Nueva Visión.

ANSOLABEHERE, Pablo (2011): Literatura y anarquismo en Argentina (1879-1919). Rosario: Beatriz Viterbo Editora.

BERTONI, Lilia Ana (2007): Patriotas, cosmopolitas y nacionalistas. La construcción de la nacionalidad argentina a fines del siglo XIX. Buenos Aires: Fondo de Cultura Económica.

BOTANA, Natalio; y GALLO, Ezequiel (1997): De la República posible a la República verdadera (1880-1910). Buenos Aires: Ariel.

CANÉ, Miguel (1899): Expulsión de los extranjeros. Buenos Aires: Imprenta de Sarrailh.

CAPPELLETTI, Ángel (1990): El anarquismo en América Latina. Caracas: Biblioteca Ayacucho.

Constitución de la Nación Argentina: texto sancionado en 1853 (2008). Buenos Aires: Lajouane.

DEVOTO, Fernando (2010): El país del primer Centenario. Cuando todo parecía posible. Buenos Aires: Capital Intelectual.

FALCÓN, Ricardo (1986-1987): "Izquierdas, régimen político, cuestión étnica y cuestión social en Argentina (1890-1912)". Anuario Escuela de Historia, segunda época, pp. 365-389.

GÁlVEZ, Manuel (1910): El diario de Gabriel Quiroga: opiniones sobre la vida argentina. Buenos Aires: Arnoldo Moen \& Hno.

GERMANI, Gino (1979): Política y sociedad en una época de transición. De la sociedad tradicional a la sociedad de masas. Buenos Aires: Paidós.

GÓMEZ TOVAR, L.; GUTIÉRREZ, R.; VÁZQUEZ, S. A.; y FALCONNET, J. A. (1991): Utopías libertarias americanas: la ciudad anarquista americana de Pierre Quiroule. Madrid: Ediciones Tueros.

Ley de Defensa Social: sancionada por el honorable Congreso de la Nación (1910). La Plata: Taller de Impresiones Oficiales.

LOMBROSO, Cesare (1895): Gli anarchici. Torino: Fratelli Bocca.

MCGANN, Thomas (1960): Argentina, Estados Unidos y el sistema interamericano 1880-1914. Buenos Aires: Editorial Universitaria.

MOYANO GACITÚA, Cornelio (1894): Notas de filosofía penal. Sobre el anarquismo. Córdoba: La Patria.

MOYANO GACITÚA, Cornelio [s.f.]: La delincuencia argentina. [S.I.]: [s.n.].

PAYÁ, Carlos; y CÁRDENAS, Eduardo (1978): El primer nacionalismo argentino: en Manuel Gálvez y Ricardo Rojas. Buenos Aires: Peña Lillo.

PELLETTIERI, Osvaldo (1980): Testimonios culturales argentinos: la década del 10. Buenos Aires: Editorial de Belgrano.

QUIROULE, Pierre (1991): La ciudad anarquista americana. Madrid: Ediciones Tuero.

RAWSON, Guillermo [s.f.]: Estudio sobre las casas de inquilinato de Buenos Aires. Buenos Aires: La Vanguardia. 
ROJAS, Ricardo (2010): La restauración nacionalista. La Plata: UNIPE.

ROMERO, José Luis (1983): El desarrollo de las ideas en la Argentina del siglo XX. Buenos Aires: Ediciones Solar.

SARLO, Beatriz (1988): Una modernidad periférica: Buenos Aires: 1920 y 1930. Buenos Aires: Ediciones Nueva Visión.

SCARZANELLA, Eugenia (2003): Ni gringos ni indios: inmigración, criminalidad y racismo en la Argentina de 1890 a 1940. Buenos Aires: Universidad Nacional de Quilmes.

SURIANO, Juan (1988): Trabajadores, anarquismo y Estado represor: de la Ley de Residencia a la Ley de Defensa Social (1902-1910).Buenos Aires: Centro Editor de América Latina.

TERÁN, Óscar (1987): Positivismo y nación en la Argentina con una selección de textos de J. M. Ramos Mejía, A. Álvarez, C. O. Bunge, J. Ingenieros. Montevideo: Puntosur.

VIÑAS, David (2004): La crisis de la ciudad señorial. Buenos Aires: Corregidor.

WEINBERG, Félix (1976): Dos utopías argentinas de principios de siglo. Buenos Aires: Solar / Hachette.

ZARAGOZA, Gonzalo (1996): Anarquismo argentino (1876-1902). Madrid: Ediciones de la Torre. 